03

\title{
Об эффекте Гуса-Хенхена при наличии возбуждения поверхностных волн в схеме Кречмана
}

\author{
(C) А.Б. Петрин \\ Объединенный институт высоких температур РАН, \\ 125412 Москва, Россия \\ e-mail: a_petrin@mail.ru \\ Поступила в редакцию 05.02.2019 г. \\ В окончательной редакции 05.02.2019 г. \\ Принята к публикации 15.03.2019 г.
}

\begin{abstract}
Рассмотрен теоретический метод исследования отражения плоского светового пучка ограниченной апертуры от плоскослоистой структуры в схеме Кречмана. Развитая теория применена к исследованию эффекта Гуса-Хенхена, возникающего при падении линейно поляризованного светового пучка с вектором поляризации в плоскости падения ( $p$-поляризованного пучка) и состоящего в том, что падающий пучок разделяется при отражении на два близких пучка той же поляризации. Обсуждена точность датчиков, основанных на этом эффекте.
\end{abstract}

Ключевые слова: поверхностные волны, поверхностные плазмоны, интегральная оптика, оптические сенсоры.

DOI: $10.21883 /$ OS.2019.10.48372.43-19

\section{Введение и постановка задачи}

В биологических, химических и физических исследованиях последнего времени большую роль играют многоканальные оптические сенсорные системы. В этих сенсорных системах для диагностики изменения показателя преломления, вызванного изменением свойств среды в тонком поверхностном слое сенсоров, широко используются поверхностные плазмонные (или плазмоннополяритонные) волны [1-4]. Поверхностные плазмонные волны распространяются вдоль поверхности металла, локализуясь вблизи его поверхности $[5,6]$, причем для возбуждения поверхностных плазмонных волн часто используется метод Кречмана [7].

В рамках схемы Кречмана [8] (рис. 1) согласование поверхностной плазмонной волны 1 на поверхности металлической пленки 2 и падающего волнового пучка 3 осуществляется с помощью стеклянной призмы 4.

Линейно поляризованный монохроматический волновой пучок падает на металлическую пленку со стороны призмы. Вектор электрического поля падающей волны $\mathbf{E}^{i}$ направлен в плоскости падения ( $p$-поляризация). Призма необходима для согласования падающей волны и поверхностной плазмонной волны по волновому числу. Волновое число поверхностной плазмонной волны больше волнового числа плоской волны в свободном пространстве той же частоты $[9,10]$. Чтобы обеспечить равенство волновых чисел падающей 3 , отраженной 5 и поверхностной плазмонной 1 волн вдоль поверхности металла (рис. 1), необходимо, чтобы падающая волна подходила к пленке из оптически более плотной среды (где волновое число больше, чем в свободном пространстве). Показатель преломления призмы и угол паде- ния подбираются таким образом, чтобы обеспечивалось равенство волновых чисел падающей и поверхностных волн. При этих параметрах в отсутствии металлической пленки (и возбуждения поверхностных плазмонов) наблюдается полное внутреннее отражение падающей волны.

Эксперименты показывают, что если угол падения соответствует согласованию по волновому числу падающей и поверхностной плазмонной волн, наблюдается резкое падение коэффициента отражения. Например, при длине падающей волны в вакууме $\lambda=633 \mathrm{~nm}$ и толщине пленки серебра, приблизительно равной $53.8 \mathrm{~nm}$ (эта величина зависит от конкретного значения показателя преломления призмы и частоты падающей волны), на-

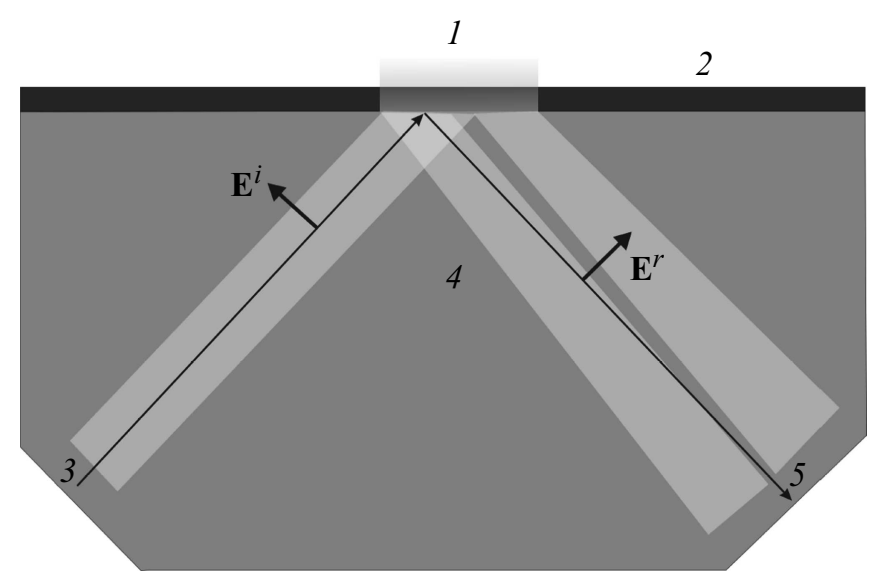

Рис. 1. Схема возбуждения поверхностной плазмонной волны 1 на поверхности металлической пленки 2 по схеме Кречмана. Падающая волна 3 в призме 4 порождает поверхностную плазмонную волну 1 и отраженную волну 5 . 
блюдается сильное изменение коэффициента отражения практически от единицы до нуля в окрестности угла падения, соответствующего согласованию свободной и поверхностной плазмонной волн. При небольшом отклонении угла падения от угла согласования коэффициент отражения возвращается практически от нуля к единичному значению.

Так как поле поверхностной плазмонной волны сосредоточено в тонком слое вблизи поверхности металла, характер отражения в схеме Кречмана сильно зависит от изменений показателя преломления в тонком слое вблизи поверхности. Именно на этом основывается широкое применение схемы Кречмана для создания различного рода датчиков, обладающих высокой чувствительностью [11]. Исследования показали, что чувствительность определяется угловой шириной минимума отражения [12], которая, в свою очередь, определяется поглощением в металле пленки. Чем меньше поглощение в металле, тем уже минимум.

Возбуждение поверхностной плазмонной волны в схеме Кречмана исследовалось для случая, когда апертура падающего светового пучка ограничена. Оказалось, что при этом отражение будет происходить не совсем так, как в случае идеально плоских волн. Так, в работе [13] было показано, что в случае ограниченной апертуры падающей волны острый минимум отражения остается, но отраженные волны имеют угловое распределение в пределах угла дифракции. Дальнейшее исследование отраженных и прошедших волн вблизи пленки [14] показало, что после отражения падающий линейно $p$-поляризованный световой пучок разделяется на две интерферирующих волны. Эти две отраженные волны, вообще говоря, отражаются с фазовым сдвигом, который зависит от влияния внешней среды на поверхностную волну. Это явление принято называть эффектом Гуса-Хенхена $[15,16]$, хотя первоначально так называли эффект пространственного разделения отраженных $s$ - и $p$-волн. Эффект используется для создания оптических сенсоров с высокой чувствительностью [17-19].

Настоящая работа посвящена исследованию эффекта Гуса-Хенхена при возбуждении поверхностных плазмонных волн на свободной границе серебряной пленки. Главные выводы допускают обобщение на общий случай возбуждения поверхностных волн в многопленочных диэлектрических структурах, таких как в работах $[20,21]$.

\section{Задача падения светового пучка ограниченной апертуры на плоскослоистую структуру}

Рассмотрим падение монохроматического светового пучка с циклической частотой $\omega$ единичной амплитуды (рис. 2) из однородного полупространства 1 (призмы), заполненного однородным изотропным диэлектриком с

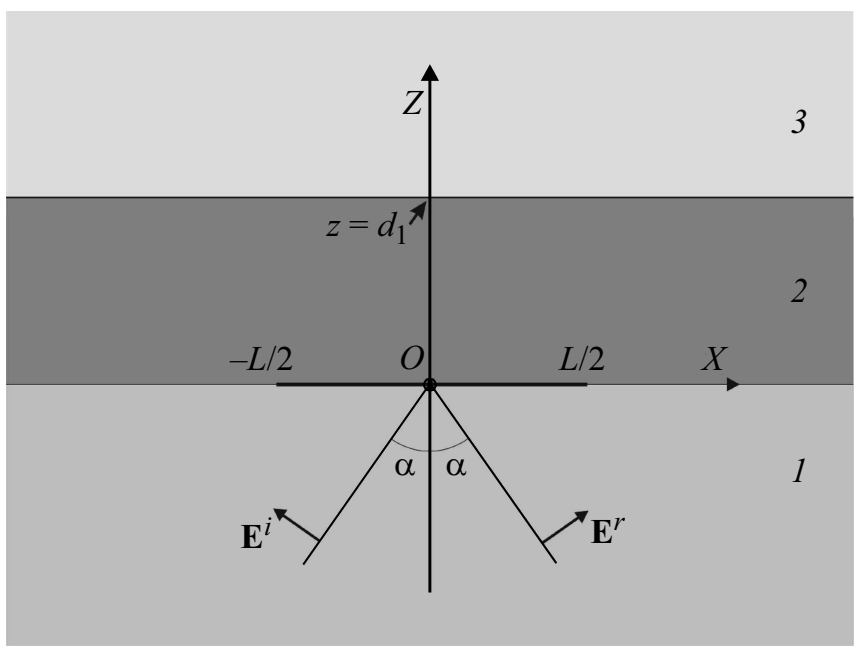

Рис. 2. Геометрия электромагнитной задачи. Плоская волна $p$ поляризации падает из призмы 1 на металлическую пленку 2, за пленкой находится вакуум 3.

показателем преломления $n_{1}$, под углом $\alpha$ к нормали на плоскую металлическую пленку толщины $d_{1}$. Комплексная диэлектрическая проницаемость металлической пленки на рассматриваемой частоте $\omega$ равна $\varepsilon_{2}$, а за пленкой находится однородное полупространство с диэлектрической проницаемостью $\varepsilon_{3}$ (свободное пространство). Введем нумерацию $j$ областей задачи; $j=1$ соответствует полупространству 1 , для которого $z<0$; $j=2$ соответствует области пленки $2(0<z<d) ; j=3$ соответствует свободному полупространству $\left(z>d_{1}\right)$. Комплексную временную зависимость примем в виде $e^{-i \omega t}$. Положим, что размер апертуры $D$ волнового фронта падающего пучка в плоскости $X Z$ таков, что пучок падает на отрезок границы раздела длины $L$ (рис. 2) под углом $\alpha$, т. е. $D=L \cos \alpha$.

Представляя компоненты электрических полей в виде фурье-разложений:

$$
\begin{aligned}
& E_{j, x}(x, z)=\frac{1}{2 \pi} \int_{-\infty}^{\infty} \tilde{E}_{j, x}(\xi, z) e^{i \xi x} d \xi \\
& E_{j, z}(x, z)=\frac{1}{2 \pi} \int_{-\infty}^{+\infty} \tilde{E}_{j, z}(\xi, z) e^{i \xi x} d \xi
\end{aligned}
$$

где фурье-образы определяются интегралами

$$
\begin{aligned}
& \tilde{E}_{j, x}(\xi, z)=\int_{-\infty}^{\infty} E_{j, x}(x, z) e^{-i \xi x} d x, \\
& \tilde{E}_{j, z}(\xi, z)=\int_{-\infty}^{\infty} E_{j, z}(x, z) e^{-i \xi x} d x,
\end{aligned}
$$


из уравнений Максвелла $[13,14]$ получим уравнения

$$
\frac{d^{2} \tilde{E}_{j, x}}{d z^{2}}+\left(\omega^{2} \mu_{j} \varepsilon_{j}-\xi^{2}\right) \tilde{E}_{j, x}=0
$$

и

$$
\frac{d^{2} \tilde{E}_{j, z}}{d z^{2}}+\left(\omega^{2} \mu_{j} \varepsilon_{j}-\xi^{2}\right) \tilde{E}_{j, z}=0
$$

Решение уравнений (1), удовлетворяющее известным граничным условиям на двух границах металлической пленки, т.е. распределения падающего и отраженного полей $\mathbf{E}_{1}^{i}(x, z)$ и $\mathbf{E}_{1}^{r}(x, z)$ в первой среде (призме), а также распределение поля, прошедшего через пленку $\mathbf{E}_{3}(x, z)$ в третью среду (свободное пространство) выражаются формулами

$$
\begin{gathered}
\mathbf{E}_{1}^{i}(x, z)=\frac{1}{2 \pi} \int_{-\infty}^{\infty}\left(\begin{array}{c}
1 \\
0 \\
-\xi / k_{1, z}
\end{array}\right) \hat{E}_{1, x}^{i}(\xi) e^{i k_{1, z} z} e^{i \xi x} d \xi, \\
\mathbf{E}_{1}^{r}(x, z)=-\frac{1}{2 \pi} \int_{-\infty}^{\infty}\left(\begin{array}{c}
1 \\
0 \\
\xi / k_{1, z}
\end{array}\right) \mathscr{R}(\xi) \hat{E}_{1, x}^{i}(\xi) e^{i k_{1, z} z} e^{i \xi x} d \xi \\
\mathbf{E}_{3}(x, z)=\frac{1}{2 \pi} \int_{-\infty}^{\infty}\left(\begin{array}{c}
1 \\
0 \\
-\xi / k_{3, z}
\end{array}\right) \mathscr{T}(\xi) \hat{E}_{1, x}^{i}(\xi) e^{i k_{3, z}(z-d)} e^{i \xi x} d \xi
\end{gathered}
$$

где $\hat{E}_{1, x}^{i}(\xi)=\int_{-\infty}^{\infty} E_{1, x}^{i}(x, 0) e^{-i \xi x} d x-$ фурье-образ $x$-составляющей электрического поля падающей волны (пучка) на границе $z=0 ; k_{j, z}=\sqrt{\omega^{2} \mu_{j} \varepsilon_{j}-\xi^{2}} ; d-$ толщина пленки; $\mathscr{R}(\xi)$ и $\mathscr{T}(\xi)$ - коэффициенты отражения и прохождения пленки бесконечной плоской волны с гармонической вариацией $e^{i \xi x}$ по оси $x$. Подробности можно найти в работах $[13,14]$.

Рассмотрим падение на пленку светового пучка с единичной амплитудой электрического поля

$$
\mathbf{E}_{1}^{i}(x, z)=e^{i\left(k_{1} x \sin \alpha+k_{1} z \cos \alpha\right)}\left(\mathbf{e}_{x} \cos \alpha-\mathbf{e}_{z} \sin \alpha\right),
$$

где $k_{1}=n_{1} \omega / c-$ волновое число в первой среде, $c$ - скорость света в вакууме, $n_{1}=\sqrt{\varepsilon_{1}}-$ показатель преломления в первой среде, $\alpha$ - угол падения пучка, $\mathbf{e}_{x}, \mathbf{e}_{z}$ - единичные векторы в направлении осей $X$ и Z. И пусть апертура пучка ограничена таким образом, чтобы при $z=0$ волна падала только на отрезок $x \in[-L / 2, L / 2]$, причем центр этого отрезка соответствует началу координат $x=0$. Тогда фурье-образ $\hat{E}_{1, x}^{i}$ падающей волны при $z=0$ можно зависать в виде

$$
\begin{aligned}
\hat{E}_{1, x}^{i}(\xi) & =\int_{-L / 2}^{L / 2} E_{1, x}^{i}(x, 0) e^{-i \xi x} d x \\
& =2 \cos \alpha \frac{\sin \left(\left(k_{1} \sin \alpha-\xi\right) L / 2\right)}{\left(k_{1} \sin \alpha-\xi\right)} .
\end{aligned}
$$

Подставляя выражение (5) в формулы (2), (3), получим падающее и отраженные поля в первой среде (при $z \leq 0)$ :

$$
\begin{aligned}
& \mathbf{E}_{1}^{i}(x, z)=\frac{L \cos \alpha}{2 \pi} \\
& \times \int_{-\infty}^{\infty}\left(\begin{array}{c}
1 \\
0 \\
-\xi / k_{1, z}
\end{array}\right) \frac{\sin \left(\left(k_{1} \sin \alpha-\xi\right) L / 2\right)}{\left(k_{1} \sin \alpha-\xi\right) L / 2} e^{i\left(\xi x+k_{1, z} z\right)} d \xi \\
& \mathbf{E}_{1}^{r}(x, z)=-\frac{L \cos \alpha}{2 \pi} \\
& \times \int_{-\infty}^{\infty}\left(\begin{array}{c}
1 \\
0 \\
\xi / k_{1, z}
\end{array}\right) \frac{\sin \left(\left(k_{1} \sin \alpha-\xi\right) L / 2\right)}{\left(k_{1} \sin \alpha-\xi\right) L / 2} \mathscr{R}(\xi) e^{i\left(\xi x-k_{1, z} z\right)} d \xi .
\end{aligned}
$$

Аналогично (из (5) и (4)) волна, прошедшая в третью среду (в область $z \geq d_{1}$ ), получится в виде

$$
\begin{aligned}
& \mathbf{E}_{3}(x, z)=\frac{L \cos \alpha}{2 \pi} \\
& \times \int_{-\infty}^{\infty}\left(\begin{array}{c}
1 \\
0 \\
-\frac{\xi}{k_{3, z}}
\end{array}\right) \frac{\sin \left(\left(k_{1} \sin \alpha-\xi\right) L / 2\right)}{\left(k_{1} \sin \alpha-\xi\right) L / 2} \mathscr{T}(\xi) e^{i\left(\xi x+k_{3, z}\left(z-d_{1}\right)\right.} d \xi .
\end{aligned}
$$

Интеграл в (6) есть интегральная сумма плоских волн, на которые можно разложить падающий пучок ограниченной апертуры. Интеграл в (7) есть соответствующая сумма отраженных плоских волн.

\section{Характер распределения отраженного поля}

Сначала было рассмотрено отражение от серебряной пленки пучка бесконечной апертуры $(L \rightarrow \infty)$ (плоской волны) по схеме Кречмана (рис. 1). Длина волны в вакууме выбиралось равной $\lambda=633 \mathrm{~nm}$. На соответствующей частоте относительные диэлектрические проницаемости серебра [22] и свободного пространства принимались равными $\varepsilon_{2}=-18.2+i 0.5$ и $\varepsilon_{3}=1$ соответственно. Было показано (см. также [21]), что для призмы с показателем преломления $n_{1}=1.6$ $\left(\varepsilon_{1}=2.56\right)$ существует оптимальная толщина пленки, равная $d=53.8 \mathrm{~nm}[21]$. Оказалось, что при такой толщине пленки при угле падения $\alpha_{\text {opt }} \approx 40.041^{\circ}$ можно добиться практически нулевого коэффициента отражения. При отклонении угла падения от $\alpha_{\text {opt }}$ на угол порядка $\pm 0.2^{\circ}$ коэффициент отражения возвращается практически к единичному значению; возникает полное отражение падающей волны [21]. В диапазоне углов резонансного взаимодействия наблюдается значительный 


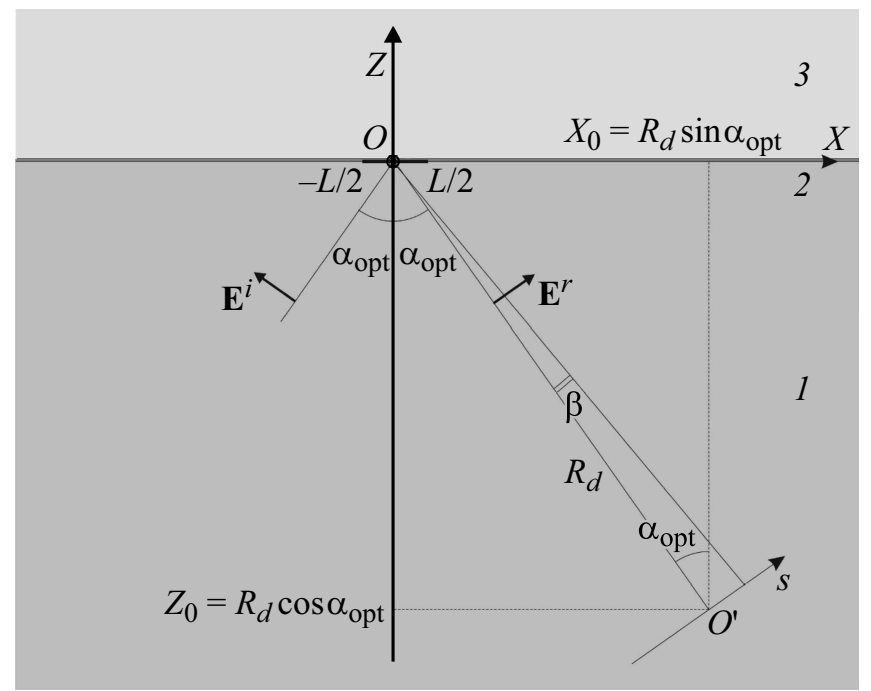

Рис. 3. Геометрия расположения оси $s$, расположенной на расстоянии $R_{d}$ от начала координат и перпендикулярная оптимальному углу отражения $\alpha_{\mathrm{opt}}$.

рост амплитуды поверхностной волны на свободной границе металла [14].

Далее было рассмотрено отражение пучка с конечным размером апертуры, описываемого формулой (7), для которой $L=200 \mu \mathrm{m}$. Распределение амплитуды отраженного поля находилось на расстоянии $R_{d}$ от центральной точки падения $x=0, z=0$ (рис. 3). Исследовалось распределение амплитуды $E_{a}^{r}$ отраженного поля вдоль оси $s$ под малыми углами (или вдоль оси $s$, перпендикулярной направлению отражения) вблизи угла оптимального (нулевого) отражения $\alpha_{\text {opt }}$. Это поле вычислялось через $x$ - и $z$-компоненты отраженного поля (7) по формуле

$$
E_{a}^{r}(s)=\left|E_{1, x}^{r}(s) \cos \alpha_{\mathrm{opt}}+E_{1, z}^{r}(s) \sin \alpha_{\mathrm{opt}}\right|,
$$

где

$$
\begin{gathered}
E_{1, x}^{r}(s)=E_{1, x}^{r}\left(x_{s}, z_{s}\right) ; \\
E_{1, z}^{r}(s)=E_{1, z}^{r}\left(x_{s}, z_{s}\right) ; \\
x_{s}=s \cos \alpha_{\mathrm{opt}}+R_{d} \sin \alpha_{\mathrm{opt}} ; \\
z_{s}=s \sin \alpha_{\mathrm{opt}}-R_{d} \cos \alpha_{\mathrm{opt}} .
\end{gathered}
$$

Или, вводя малый угол $\beta$ отклонения от $\alpha_{\mathrm{opt}}$, такой что $s=R_{d} \operatorname{tg} \beta$, можно ввести зависимость вдоль оси $s$ от этого угла в виде

$$
\begin{aligned}
E_{a}^{r}(\beta)= & \mid E_{1, x}^{r}\left(R_{d} \operatorname{tg} \beta\right) \cos \alpha_{\mathrm{opt}} \\
& +E_{1, z}^{r}\left(R_{d} \operatorname{tg} \beta\right) \sin \alpha_{\mathrm{opt}} \mid .
\end{aligned}
$$

Поле $E_{a}^{r}(\beta)$ убывает при увеличении расстояния $R_{d}$ пропорционально $R_{d}^{-1 / 2}$. Поэтому, чтобы иметь представление о распределении интенсивности отраженной волны, приходящей на ось $s$ на расстоянии $R_{d}$, были вычислены зависимости $I(\beta)=\left(E_{a}^{r}(\beta)\right)^{2} R_{d}$ для различных значений $R_{d}$ (рис. 4 ). Отметим, что величина $E_{a}^{r}$ в наших вычислениях находилась в предположении, что амплитуда падающей волны равна единице (при желании, зная амплитуду напряженности электрического поля падающей волны, $I(\beta)$ и $R_{d}$, из зависимостей рис. 4 нетрудно вычислить точные распределения интенсивности поля как функции $s$ ).

Важно, что если зафиксировать некоторое $R_{d}$, то из зависимостей рис. 4 следует, что в окрестности угла отражения $\alpha_{\text {opt }}$ в плоскости $s$ будет два главных максимума интенсивности отраженной волны, которые можно считать двумя отраженными пучками. Эти отраженные пучки имеют примерно одинаковую угловую ширину и практически нулевую интенсивность посередине. Ноль распределения несколько смещается при изменении $R_{d}$ из-за того, что фазовый центр отражения смещен относительно начала координат. Это связано с несимметричным отражением ограниченного пучка при возбуждении поверхностной плазмонной волны [14].

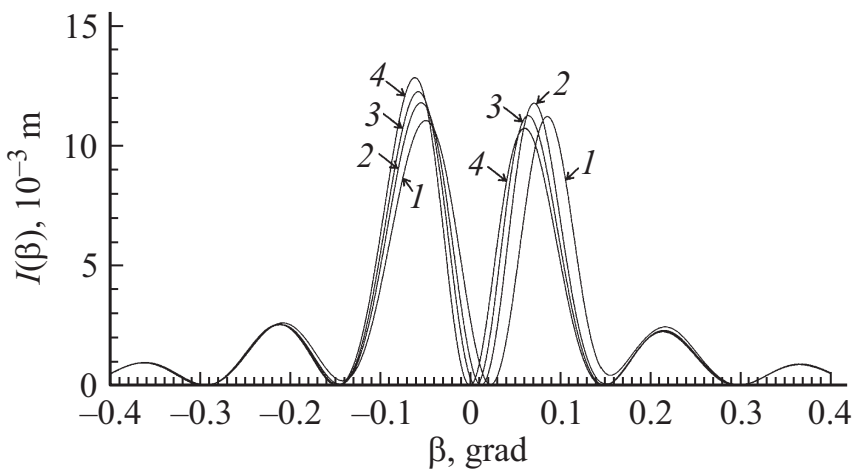

Рис. 4. Распределения величины $I(\beta)=\left(E_{a}^{r}\right)^{2} R_{d}$ как функции угла отклонения $\beta$ при разных значениях $R_{d}$. Кривая 1 - для $R_{d}=0.1 \mathrm{~m}, 2-R_{d}=0.2 \mathrm{~m}, 3-R_{d}=0.4 \mathrm{~m}$ и $4-R_{d} \rightarrow \infty$.

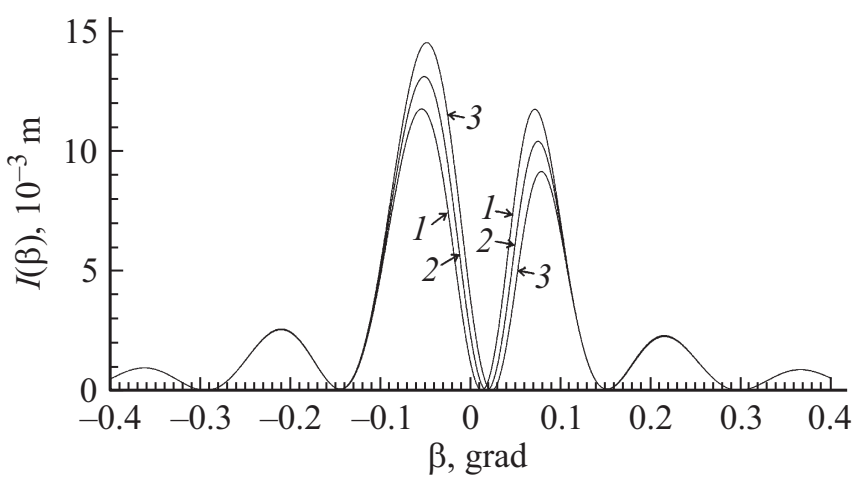

Рис. 5. Распределения величины $I(\beta)=\left(E_{a}^{r}\right)^{2} R_{d}$ как функции угла отклонения $\beta$ на расстоянии $R_{d}=0.2 \mathrm{~m}$ при различных толщинах пленки воды $h_{w}$ на свободной поверхности серебряной пленки. Кривая $1-$ для $h_{w}=0 \mathrm{~nm}, 2-h_{w}=0.1 \mathrm{~nm}, 3-$ $h_{w}=0.2 \mathrm{~nm}$. 
Если показатель преломления свободного пространства вблизи границы будет изменяться, то при прочих равных условиях нуль распределения $I(\beta)$ будет смещаться. На измерении этого смещения основаны экспериментальные методики, которые используются в датчиках на эффекте Гуса-Хенхена [23,24].

Взгляд на возбуждение поверхностных волн в схеме Кречмана как на разделение отраженной волны на два пучка, сопровождающееся эффектом ГусаХенхена, является важной идеей для разработки высокочувствительных сенсоров, реагирующих на изменения показателя преломления среды вблизи свободной поверхности пленки, сопровождающихся смещением нуля суммарной отраженной волны. Для измерения указанного смещения нуля дифракционной картины отраженной волны применяются высокоточные экспериментальные методики, разработанные для сенсоров на эффекте Гуса-Хенхена [24] и в технике оптического пинцета [25,26]. Особое значение имеют дифференциальные методы измерения, использующие квадрантные фотодиоды (quadrant photodiodes). Использование квадрантных фотодиодов и дифференциальных методов измерения [24] дает возможность не только избавиться от экспериментальных ошибок, связанных с нестабильностью амплитуды падающей волны, но и от влияния температурной нестабильности и акустических вибраций экспериментальной установки.

Чтобы продемонстрировать чувствительность дифференциальных методов измерения, были вычислены распределения интенсивности отраженной волны, точнее, пропорциональной ей величины $I(\beta)$, от угла $\beta$ на расстоянии $R_{d}=0.2 \mathrm{~m}$ от начала координат для трех случаев (рис. 5). Первый случай (кривая 1 на рис. 5) это случай, когда на свободной поверхности пленки ничего нет (то же, что и кривая 2 на рис. 4). Второй случай (кривая 2 на рис. 5), когда на свободной поверхности серебряной пленки находится пленка воды с показателем $n_{w}=1.33$ и толщиной $0.1 \mathrm{~nm}$. (Понятно, что пленку воды такой толщины нельзя считать однородной средой, но для оценок влияния дополнительной пленки на дифракционную картину это приемлемо.) Наконец, третий случай (кривая 3 на рис. 5), когда на свободной поверхности находится такая же пленка воды, но толщиной $0.2 \mathrm{~nm}$.

Из рис. 5 видно, что при ничтожных изменениях интегрального показателя преломления поверхностного слоя на свободной поверхности серебряной пленки сенсора происходит не только заметный сдвиг нуля дифракционной картины, но и появляется заметная асимметрия распределения, которая с легкостью будет обнаружена квадрантным фотодиодом с дифференциальной схемой включения, находящимся в плоскости наблюдения $s$ (рис. 3).

\section{Заключение}

Теоретическое рассмотрение отражения ограниченного светового пучка $p$-поляризации с однородным распределением амплитуды по его апертуре в схеме Кречмана позволило изучить разделение отраженной волны на два расходящихся интерферирующих пучка той же поляризации. Вычисления прямо продемонстрировали большую чувствительность сенсоров на основе рассмотренного эффекта Гуса-Хенхена к изменению показателя преломления в тонком слое вблизи свободной поверхности сенсора. Подчеркнута перспективность применения квадрантных фотодиодов в качестве датчиков смещения дифракционной картины отраженной волны. Представленная теория может быть полезна при разработке современных оптических многоканальных биологических, химических и физических сенсорных систем.

\section{Конфликт интересов}

Автор заявляет, что у него нет конфликта интересов.

\section{Список литературы}

[1] Homola J., Yee S.S., Gauglitz G. // Sensors and Actuators B. 1999. V. 54. P. 3.

[2] Barnes W.L., Dereux A., Ebbesen T.W. // Nature. 2003. V. 424. P. 824.

[3] Homola J. // Chem. Rev. 2008. V. 108. P. 462.

[4] Spoto G., Minunni M. // J. Phys. Chem. Lett. 2012. V. 3. P. 2682.

[5] Raether H. Surface Plasmons. Berlin: Springer-Verlag, 1988. $353 \mathrm{p}$.

[6] Barnes W.L. // J. Opt. A: Pure Appl. Opt. 2006. V. 8. P. S87.

[7] Kretschmann E., Raether H.Z. // Naturforsch. A. 1968. V. 23. P. 2135.

[8] Piliarik M., Homola J. // Opt. Express. 2009. V. 17. N 19. P. 16505.

[9] Liedberg B., Nylander C., Lundstrom I. // Sensors and Actuators. 1983. V. 4. P. 299.

[10] Liedberg B., Nylander C., Lundstrom I. // Biosensors \& Bioelectronics. 1995. V. 10. P. i-ix.

[11] Garabedian R., Gonzalez C., Richards J. et al. // Sensors and Achrators A. 1994. V. 43. P. 202.

[12] Yeatman E.M. // Biosensors \& Bioelectronics. 1996. V. 11. P. 635.

[13] Петрин А.Б. // Опт. и спектр. 2018. Т. 125. № 3. С. 375.

[14] Петрин А.Б. // Опт. и спектр. 2018. Т. 125. № 6. С. 830.

[15] Goos F., Hänchen H. // Ann. Phys. 1947. V. 1. P. 333.

[16] Renard R. // J. Opt. Soc. Am. 1964. V. 54. P. 1190.

[17] Yin X., Hesselink L. // Appl. Phys. Lett. 2006. V. 89. P. 261108.

[18] Wan Y., Zheng Z., Zhu J. // J. Opt. Soc. Am. B. 2011. V. 28. N 2. P. 314.

[19] Parks A.D., Spence S.E. // Appl. Opt. 2015. V. 54. N 18. P. 5872.

[20] Петрин А.Б., Вольпян О.Д., Сигов А.С. // Опт. и спектр. 2017. T. 123. № 5. С. 786. 
[21] Петрин А.Б., Вольпян О.Д., Сигов А.С. // ЖТФ. 2018. Т. 88. № 3. C. 433.

[22] Новотный Л., Хехт Б. Основы нанооптики. / Под ред. Самарцева В.В. М.: Физматлит, 2009. 484 с.

[23] Tao N.J., Boussaad S., Huang W.L., et al. // Rev. Sci. Instrum. 1999. V. 70. N 12. P. 4656.

[24] Zhang H.Q., Boussaad S., Tao N J. // Rev. Sci. Instrum. 2003. V. 74. N 1. P. 150.

[25] Neuman K.C., Block S.M. // Rev. Sci. Instrum. 2004. V. 75. N 9. P. 2787.

[26] Nugent-Glandorf L., Perkins T.T. // Opt. Letters. 2004. V. 29. N 22. P. 2611. 